\title{
Synthesis of Antibacterial Coating Using Chitosan, Polyethylene Glycol and Silver Nanoparticles and Investigation of Their Antibacterial Properties
}

\author{
Saba Qureyshi ${ }^{1}$, Zaman Sajid ${ }^{2, *}$ \\ 1. School of Chemical and Material Engineering SCME, National University of Sciences and Technology NUST, \\ Islamabad, Pakistan \\ 2. Department of Process Engineering, Faculty of Engineering and Applied Science, Memorial University of \\ Newfoundland, St. John's, NL, A1B 3X5, Canada \\ Email:sabaqureyshi@gmail.com;zaman.sajid@mun.ca(Correspondingauthor)
}

Received: 7 October 2020; Accepted: 29 October 2020; Available online: 10 May 2021

\begin{abstract}
Chitosan/ Polyethylene glycol and silver nanoparticles based antibacterial coating has been synthesized and applied to cotton cloth using sonochemical technique. In addition to the synthesis of nanoparticles using Pyrus seed extract (Green synthesis), Chitosan and PEG compound has also been used to develop the coating in this research. The coating obtained with this compound possesses good antibacterial properties and results. The study shows that the coated fabrics and silver nanoparticles show highly potent antibacterial activity towards gram negative and gram-positive bacteria. A comparison of coating with single and multiple components is studied; specifically, a comparison of pure chitosan and polyethylene glycol coating with their blend is studied. Agar plate test is performed against pseudomonas aeruginosa, Acinetobacter baumannii and methicillin-resistant staphylococcus aureus (MRSA), and the proposed process is helpful in healthcare industry and specified applications.
\end{abstract}

Keywords: Silver nanoparticles; Chitosan; Antibacterial coating.

\section{Introduction}

Silver has various properties at Nano level and a study of such properties can help to explore more about its applicability in various field of science and engineering[1].The scope of silver nanoparticles is not confined to medical industry but it has also been under research interest for surface enhanced Raman scattering, food and packaging, additives, catalysis, textile and anti-microbial [2-5]. The antibacterial activity is performed by silver nanoparticles by attaching them to the cell wall of microbes causing disturbance in its permeability and in its respiration [3]. Interaction of nanoparticles with DNA through cell wall penetration is also a unique characteristic. Since it releases silver ion; it is more effective in antimicrobial activities [6]. To avoid microbial contamination; silver nanoparticles have been incorporated in polymers for their applications in wound/burn dressings, dental material, catheters, as well as in medical Implants and devices [7-10]. Due to various applications of silver nanoparticles, various methods have been advised for its production [11-15]. In chemical synthesis of silver nanoparticles, citrate and sodium borohydride are the commonly used reducing agents [16-18]. Since chemical methods can be subjected to toxic materials released which in turn can cause environment issues; biological method of synthesis of silver nanoparticles has been developed. In this work silver nanoparticles were synthesized through green route using Pyrus seed extract for the first time. To the best of authors' knowledge such novel route has never been adopted earlier.

As the experimentation is going on antibacterial coatings it develops many research gaps which needs to be filled. Over the time, the knowledge on functional coatings and synthetic coatings has been evolved and is becoming a research focus. With the upsurge in health responsiveness, many human beings are concerned about their education and protection from pathogens. Now it has become special concern to protect the wearer from pathogens than just protecting fibers [19].

Research has shown that higher possibilities for the places for pathogens to grow are our homes [20-21]. The textile materials accelerate the growth of bacteria. Some coatings accelerate this process of microorganisms' growth [22]. A study proves that how ordinary fibers like wool and cotton are vulnerable to bacterial growth and the growth of dust fibers hold oxygen, nutrients and water. Bacteria is commonly found in home closets, beds, curtains, carpets, pillows, linens and foam mattress [23].

Another study shows that owing to growing population and different kinds of diseases, researchers are focusing on developing coatings for textile which can be used in hospitals [24]. Patients, employees, visitors are vulnerable 
at cross transmission of diseases. Bacteria are tiny creatures/microbes which cannot be seen through naked eyes; however, they affect human life. Bacteria need food and water to metabolize them for their survival [25]. Water can be taken from fabrics, perspiration of body and humidity from air while their food is usually taken from dust and skin cells. Bacteria are unicellular organisms which are found as cocci, rod and spiral; they live in pairs, chains or clusters [26]. There are two kinds of bacteria: gram positive and gram-negative bacteria respectively; used for antibacterial testing and they are identified through Gram-stain test [27]. Cotton fabric has poor resistance towards microorganisms, so the use of antibacterial coating becomes important in this case [28-30].

All anti-bacterial coatings have main function generally; that is to reduce the bacterial spread either inhibiting them or killing them by their contact with the surface of fabric [31-32]. In earlier times, salts of different metals especially copper and chlorinated waxes were used to protect the textile. In late 1950s US department of Agriculture researched on the environmental impact of using antibacterial coatings on textile [33]. The conventional antibacterial agents that are commonly used are N-helamines, Quaternary ammonium compounds, Phenols, Chitosan and metallic salts [34-35]. Research is also being performed to develop a device which could coat nanoparticles in situ to the fabric. They worked with copper oxide and zinc oxide Nano particles. The coated fabric can be used as wound dressings, hospital beddings and medical uniforms for the staff [35-36].

Anna and colleagues developed reactor to introduce nanoparticles of copper oxide in the fabric using ultrasonic cavitation [37]. They developed model of simulation by varying pressure. The coated fabric with this method shows good antibacterial properties [38]. Ahoran and co-researchers developed a system to coat metal oxide nanoparticles of zinc, magnesium and copper respectively on fabric using sonochemical method. The sole purpose of this fabric was its use in outdoor clothing [39]. In the hospital, microbes are continuously spread due to the patients contact with the bed sheets hospital acquired diseases. Research has shown that the effective antibacterial textile is the need to prevent the hospital acquired diseases. They developed one step sonochemical method to coat the fabric, which was the simultaneous method of coating Chitosan and Zinc Oxide nanoparticles. The coated fabric also sustained multiple laundry cycles [40]. In another study researchers studied the coating of silver nanoparticles on the fabric using sonic irradiations. Their study revealed that both gram positive and negative bacteria show excellent antibacterial activity [41]. Purwarand and colleagues studied that synthetic fiber is also the main focus in textile industry [42]. Chitosan a natural polymer having antibacterial effects, is a polysaccharide and is derived from Chitin, showing chelating, film forming and polycationic properties due to its hydroxyl and active amino groups [43].

In the current study, Chitosan, polyethylene glycol and silver nanoparticles (AgNPs) are used to coat the cotton fabric through the sonochemical method. The novelty in the process introduced in this research comes from the fact that it is an efficient and only one step process, and hence eliminates the need for complex arrangements i.e. the coating combination of different compounds with silver nanoparticles and with each other respectively, for the whole process.

\section{Materials and methods}

\subsection{Materials}

Chitosan flakes and polyethylene glycol (PEG) 20000 were obtained from Sigma Aldrich. Acetic acid, Silver nitrate $\left(\mathrm{AgNO}_{3}\right)$ were of analytical grade and taken from chemical laboratory. Solutions which were used in experiments were prepared in deionized water.

\subsection{Methods}

The experiment included five variations of treatment - four finishes and a control with no added finish. The fiber was cut into five squares in which two were treated with coating having varying concentrations of silver nanoparticles and other two were coated with polyethylene glycol and chitosan respectively. Further these samples were used for testing.

Chitosan was dissolved in water by adding acetic solution in mixture and this mixture was kept on stirring until it dissolved completely. Polyethylene glycol 20000 was used in this coating, which is dissolved in distilled water. Silver nanoparticle solutions were prepared for concentrations of $0.01 \%$ and $0.1 \%$. All prepared solutions were added in a separate beaker and were allowed it stir for 5 minutes. After that pieces of cotton were put in the beaker and sonication was being done for 20 minutes for the uniform coating on all over the fabric samples. Same chitosan and PEG mixture was used for coating on fabric and is subjected to sonication for the same time period. Small square samples used for antibacterial analysis were taken from textile. Treated samples were dried for 20 minutes at $70^{\circ} \mathrm{C}$. The experimental setup is shown in Figure 1. 


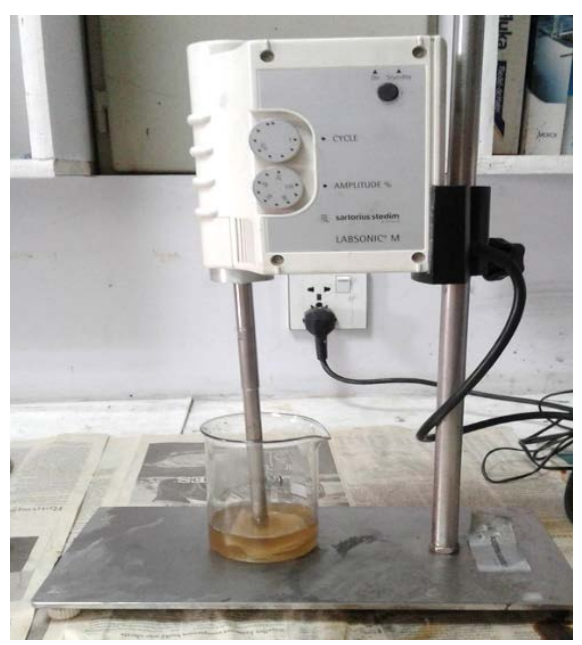

Fig 1. Coating through sonochemical method in the laboratory

\section{Characterization}

The morphology of the samples surfaces fabric and nanoparticles were examined by scanning electron microscope (SEM) images using a JEOL-6490A-JSM. The purpose of using SEM was to examine morphology of silver nanoparticles and to examine if the applied coating on textile is effective. The SEM was used to scheme an image of each sample (i.e. with only chitosan and polyethylene and silver nanoparticles at different concentrations, no treatment) to inspect visually any changes in the sample surface, across the 5 fabrics and nanoparticles. For preparing samples, the samples were held using conductive pressure sensitive glue and were sputtered with a gold coating to secure an appropriate image.

To examine and analyze the quality and type of compound which has been produced during the reaction, an Xray diffraction (XRD) was carried out. Using dried powder sample of silver nanoparticles was prepared for the STOE Theta/theta diffractometer which was being operated on $40 \mathrm{kV}$ with current of $30 \mathrm{~mA}$ and radiations of $\mathrm{Cu}$ $\mathrm{K} \alpha$ between ranges of $-10^{\circ}$ to $168^{\circ}$. Infrared spectroscopy technique was used to identity the chemical group in the material. Different chemical compounds absorb infrared radiation at frequencies corresponding to their own molecular vibrational frequencies. Then samples were placed in Fourier-transform infrared spectroscopy (FTIR) for analysis which was carried out in ranges of $4000-400 \mathrm{~cm}^{-1}$ with resolution of $4.0 \mathrm{~cm}^{-1}$ and the spectrometer model used was Perkin Elmer spectrometer FTIR spectrum 100.

The antibacterial experiments were made on textile specimens consuming the AATCC Standard Test Method 100.Pseudomonas aeruginosa, acineto bacterbaumannii and methicillin-resistant staphylococcus aureus (MRSA) were used to investigate the antibacterial characteristics of samples under study. The specimens were disinfected under a UV source previously they were there bare to the microbes. In this study, strains of bacteria were used as alternatives for gram negative and positive bacteria. Prepared colonies of bacteria were taken for experimental purpose. The bacteria grown; was diluted with sterile saline.

\section{Results and discussion}

\subsection{Scanning Electron Microscopy (SEM)}

SEM images were taken for topographical analysis of coated cotton samples. SEM micrograph was used to evaluate the surface morphology of both the untreated and Cotton fabric with AgNPs, Chitosan and PEG these images show the morphological changes due to the coating on cotton fabric containing AgNps, Chitosan and PEG. These figures show concentrated particles at some locations on the fabric and the modification in the fiber surface. The results of the analysis show that there was graphic alteration among the textile surfaces of the treated and untreated samples. At magnification, surfaces of samples treated with either chitosan polyethylene glycol and silver nanoparticles looked very smooth and uniform. On pictorial inspection, the sample with no treatment displays roughness and cracks on its exterior. The analysis also shows that the surface of the treated textile is coarser than that of untreated textile. There are morphology changes due to the coating of chitosan, PEG and AgNPs. Figure 2 to 4 show the scanning electron microscopy for cotton textile before and after coating with chitosan/polyethylene glycol/AgNPs. The formation of smooth layer on fabric surface is quite visible.

In Fig 2 and 3, the silver nanoparticles are seen clearly bright and visible spherical nanoparticles on the surface of Nano composite coating. 
Figure 4 shows smooth coating of Nano composite and no bridging of material has been seen. Homogenous coating is appeared in the image.

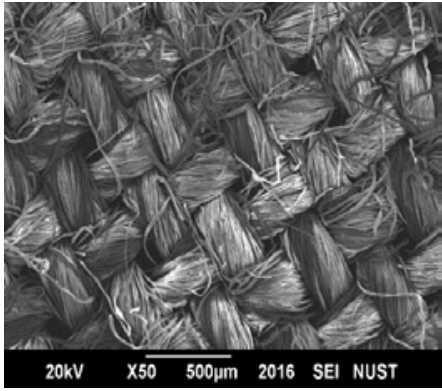

(a)

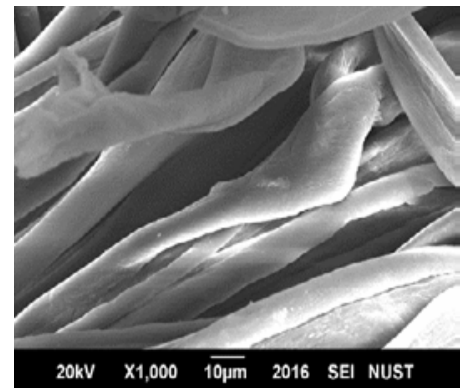

(b)

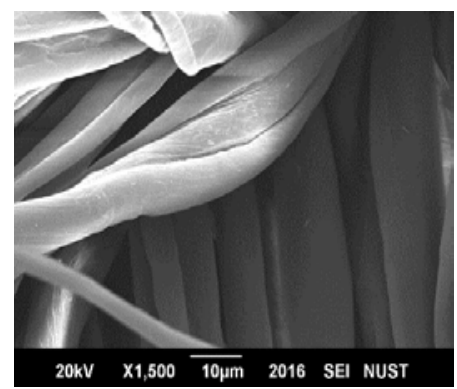

(c)

Fig. 2. SEM images of (a) untreated cotton (b) chitosan treated (c) PEG treated textile

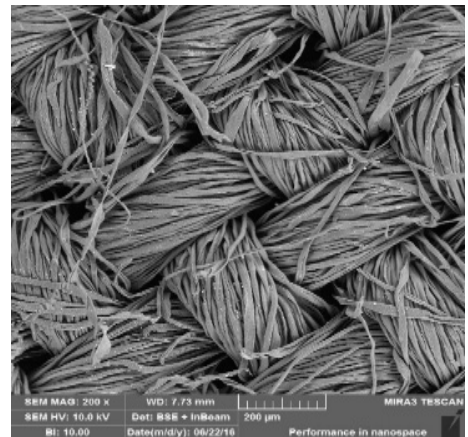

(a)

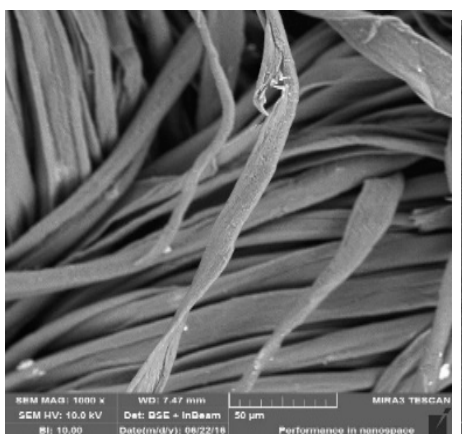

(b)

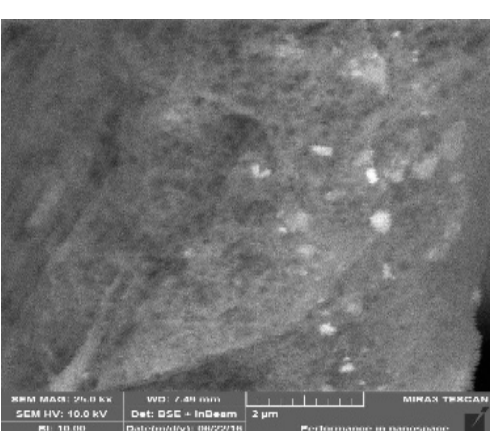

(c)

Fig 3. SEM images of sample with 0.01\% AgNPs in PEG/Chitosan coating, (a), (b) and (c) images are taken atdifferent magnifications

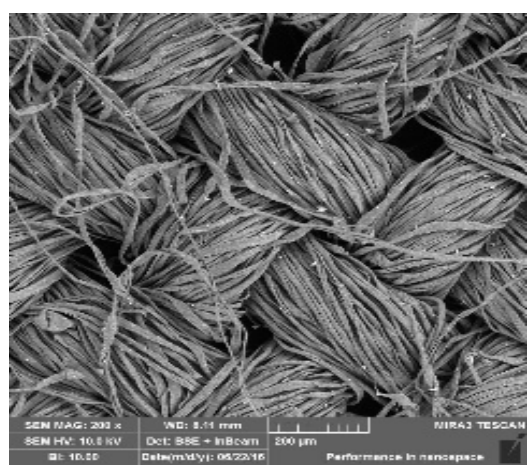

(a)

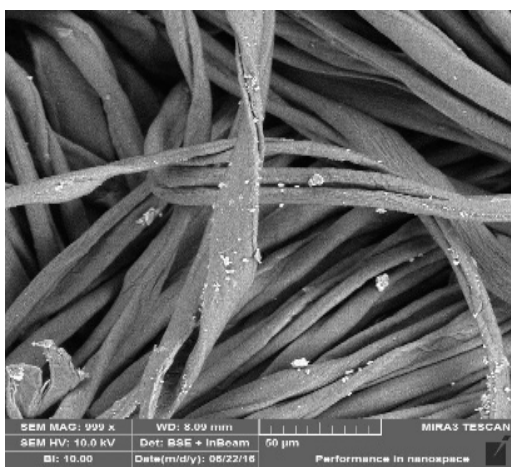

(b)

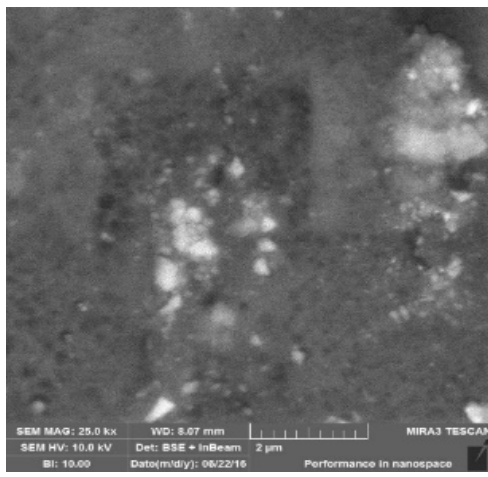

(c)

Fig 4. Sample with 0.1\% AgNPs in PEG/Chitosancoating, (a), (b) and (c) images are taken at different magnifications

\subsection{X-Ray Diffraction (XRD)}

The XRD studies show numbers of Bragg's reflections. Figure 5- 6 show the XRD graphs.

The XRD pattern for sample 1of $0.1 \%$ concentration and sample 2 of $0.01 \%$ concentration of silver nanoparticles indicates successful deposition of Ag NPs on the as prepared samples. The XRD graphs show a prominent PEG/Chitosan peak at 2 theta of 23.20 degree. The peaks for silver nanoparticles can be seen at 2 theta of 43.70 and 64.06 degree referring to (200) and (220) planes respectively. This indicates the face centered cubic structure of silver nanoparticles. Sharp peaks show high crystalline nature of deposited silver nanoparticles. Intensity of the deposited AgNPs increased with theincreasing concentration of the deposited AgNPs which shows the highly crystalline morphology as well as homogeneous nature of the reaction. The identified peaks show that the samples are composed of silver nanoparticles and PEG/Chitosan being present inside without any other identified impurity found in the pattern. Therefore, it is evident that silver nanoparticles are present in Ag/Chitosan/PEG deposited samples. 


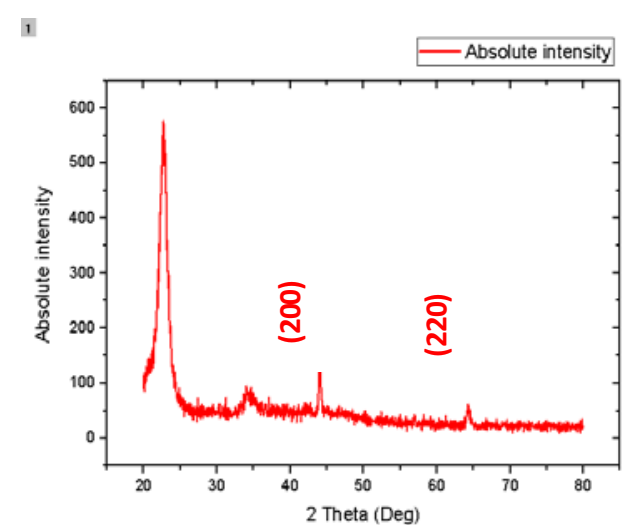

Fig 5. X-Ray Diffraction Pattern of coating sample with PEG, Chitosan and 0.01\%AgNPs

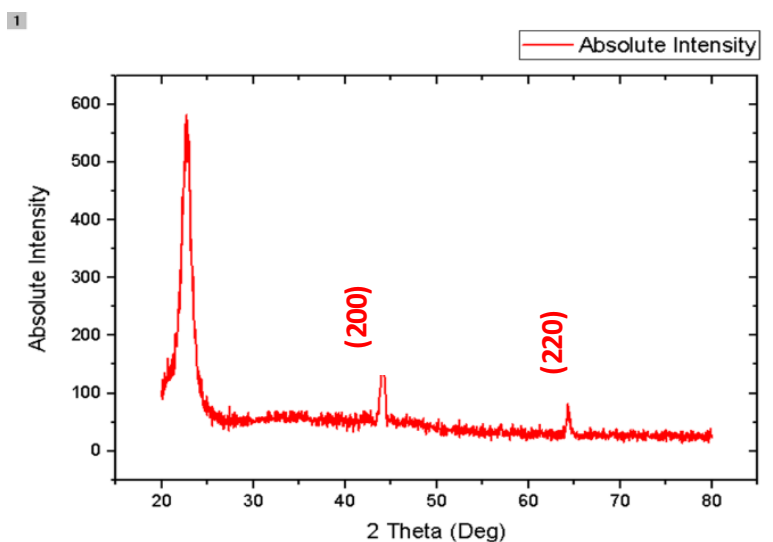

Fig 6. X-Ray Diffraction Pattern of coating sample with PEG, Chitosan and $0.1 \%$ AgNPs

\subsection{Fourier Transform Infrared Spectroscopy (FTIR)}

The FTIR analysis of silver nanoparticles and coated cotton fabrics were performed and the results are shown in Figures $7-10$.

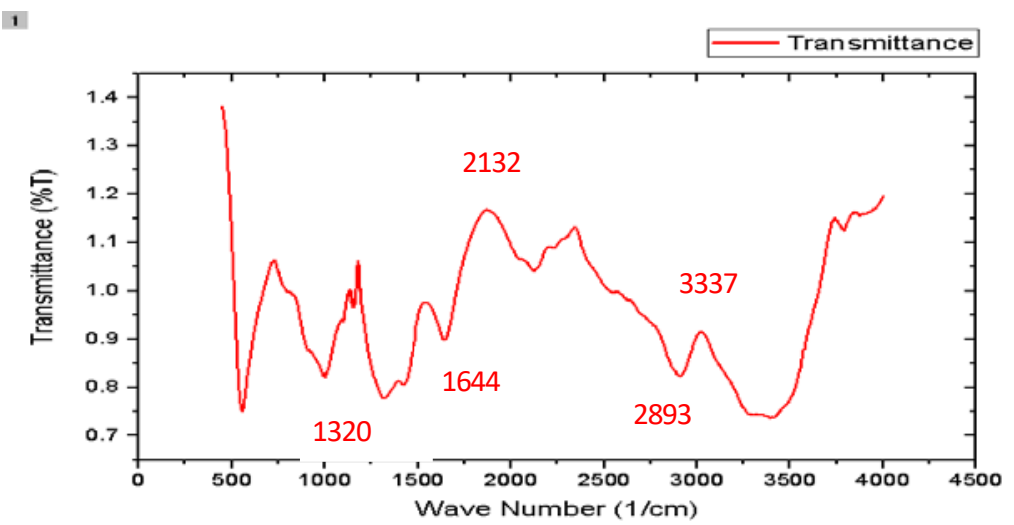

Fig 7. Pure PEG coated on fabric

As shown in fig 7, the broader peak shows the presence of -OH group around wave number 3337. The peak at wave number 2893 refers to carboxylic group with medium bonding and stretching vibration. Peak at 2132 indicates alkyne group. The peak at wave number 1644 shows Alkene $\mathrm{C}=\mathrm{C}$ group. The peak at wave number 1320 refers to carboxylic $\mathrm{CH}_{3}$ group showing stretching vibration.

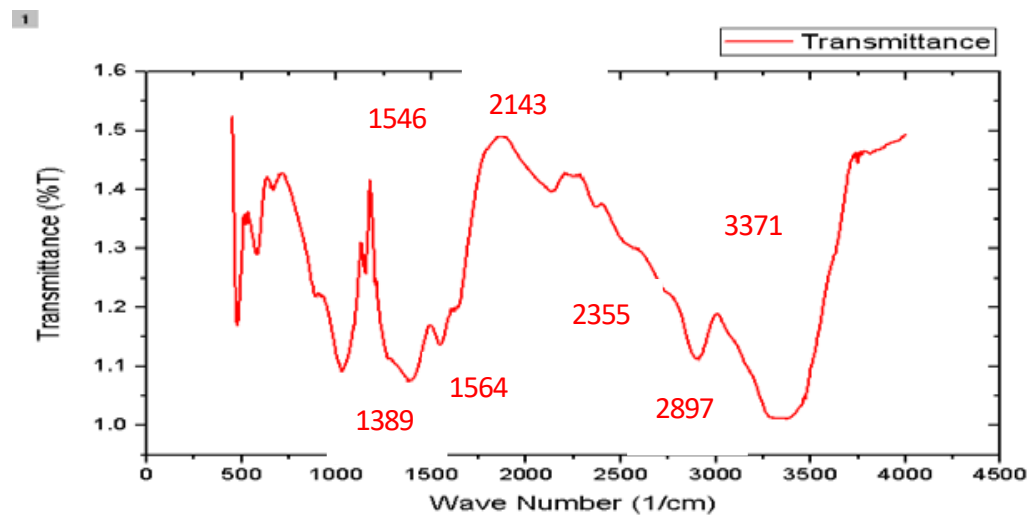

Fig 8. Pure Chitosan coated on fabric

In Figure 8, the broad peak around wave number 3371 shows Alcohol group (O-H) with stretching vibration. The peak at wave number 2897 refers to alkyl group with sp3 hybridization. Peaks at wave number 2355 and 2143 
are nitrile and alkyne groups respectively. The peak at wave number 1546 shows strong bond with stretching vibration of aromatic compound. The broad peak at around wave number 1389 refers to $\mathrm{CH}_{3}$ group with deformation vibration.

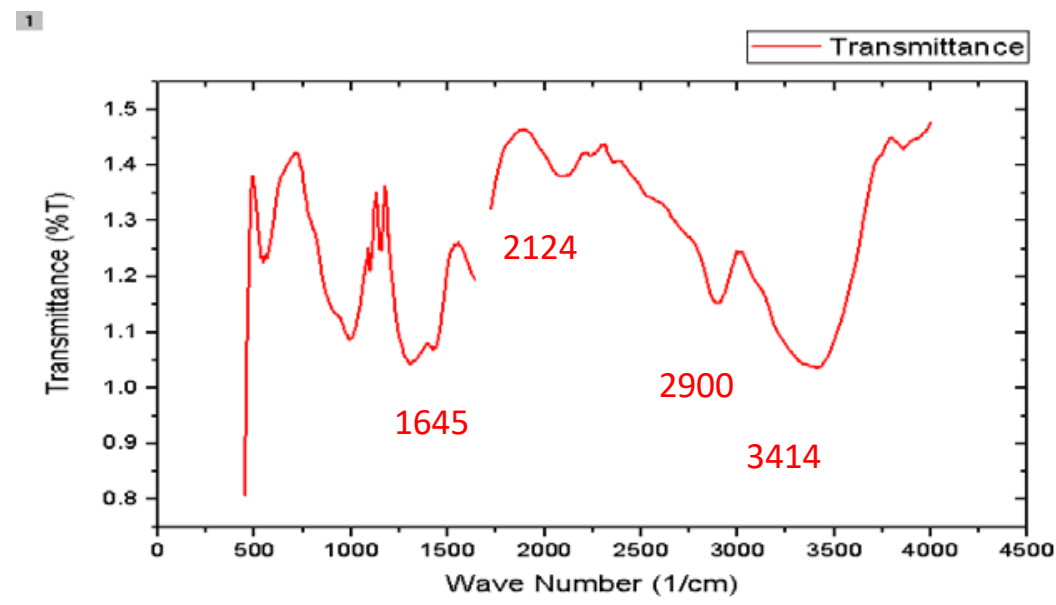

Fig 9. Chitosan/PEG/ AgNPS with $0.01 \%$ conc. is coated on fabric

Referring to Fig 9, the broad peak around wave number 3414 shows Alcohol group (-OH) with strong bond and stretching vibration. The peak at wave number 2900 refers to alkyl group with sp3 hybridization. The slightly broad peak at wave number 2124 refers to Alkyne group with stretching vibration. The strong peak at around 1645 refers to alkene $(\mathrm{C}=\mathrm{C})$ group with stretching vibration.

As shown in Figure 10, the broad peak around wave number 3429 shows Alcohol group $(\mathrm{O}-\mathrm{H})$ with stretching vibration. The peak at wave number 2898 refers to alkyl group with sp3 hybridization. The peak at wave number 2350 refers to nitrile group. The peak at wave number 1180 indicates amine group.

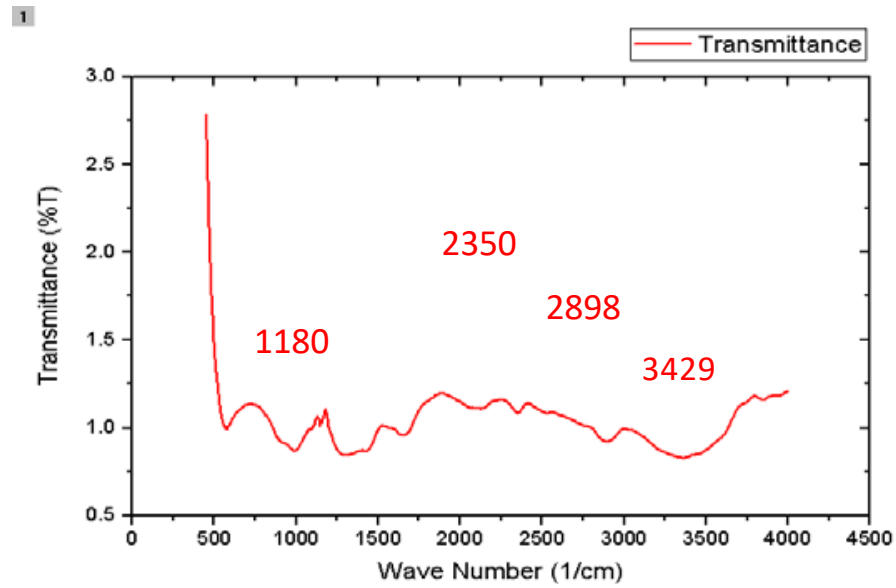

Fig 10. Chitosan/ PEG/ AgNPS with 0.1\% conc. is coated on fabric

\subsection{Agar plate Test}

The treated fabric specimens with different amount of AgNPs in coating to create antibacterial compounds shows significant biocidal activities with varying degrees of zones of inhibitions depending on silver nanoparticles concentration. The synthesis and the testing of silver nanoparticles used in this research have been done in earlier work. [44]. It is clear from the Fig. 11 that the Chitosan/ PEG/ AgNPs treated cotton fabric effective against MRSA, P. Aeruginosa and A. Baumannii with varying zone of inhibition. Results indicate that for both coating and particle with no bacterial presence is observed which is considered acceptable antimicrobial activities of a treated fabric. These results confirm that the fabric has antibacterial activities against both gram positive (MRSA) and gram negative (P. Aeruginosa and A. Baumannii) bacteria respectively. These three organisms were chosen for the biocidal activities because they are the most commonly used organisms for testing purposes.

Fig 11 shows antibacterial results. 

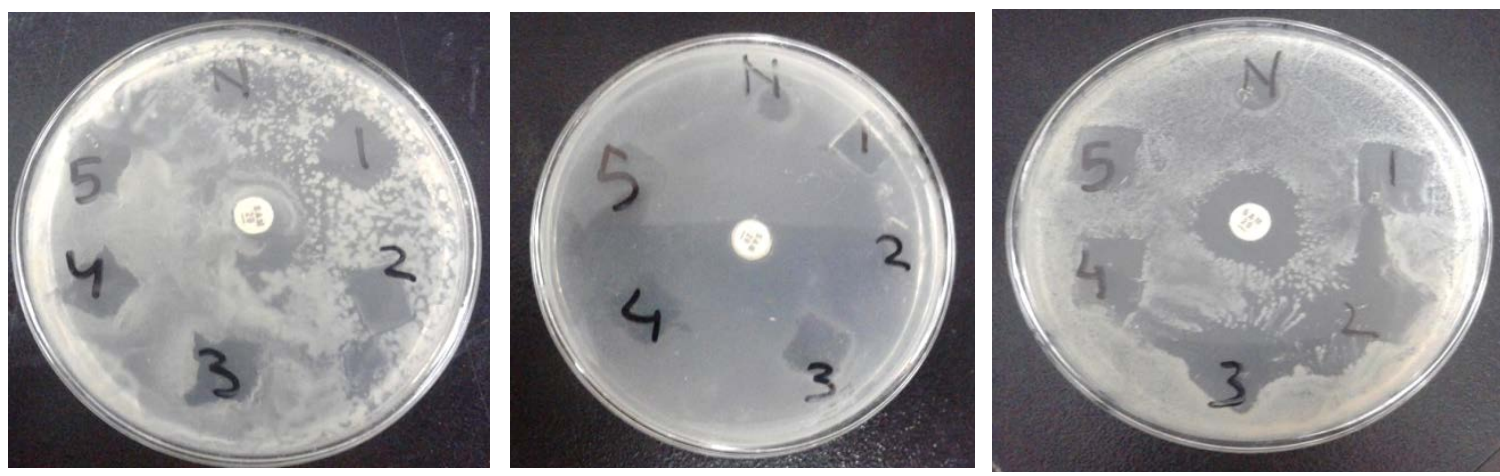

Fig11. Zone of inhibition of silver nanoparticles and four samples of coating against(A) Acinetobacter baumannii (B) Methicillin-resistant Staphylococcus aureus (C) Pseudomonas aeruginosa. (0) is control present in center (N) is AgNPs prepared with conc. of $100 \mathrm{mg} / \mathrm{L}$ (1) coating with $0.1 \%$ silver nanoparticle, chitosan and polyethylene glycol (2) coating with $0.01 \%$ silver nanoparticles, chitosan and polyethylene glycol (3) coating of chitosan (4) coating of polyethylene glycol (5) blank.

\section{Conclusion}

This research introduces a novel process which is less costly, more convenient and provides durable antimicrobial property for everyday clothing, hygiene products, and textile. The proposed process provides a feasible, easy, and robust process that can be carried out in the textile processing plants, creating more durable properties for the fabric. One of the benefits of adding PEG to the chitosan was to enhance surface properties since it is biocompatible, so keeping in mind its biological and environmental impact it is used along with PEG, which also possess antibacterial properties and also gives a good final texture making it soft and considering the pros and cons of PEG, it provides good stretching and flexible properties to fabric. Silver is proven antibacterial agent through centuries and silver nanoparticles used in this coating are to enhance its antibacterial properties. All tested samples indicate clearing in the antibacterial assays. The use of biodegradable polymers makes it possibleto create robust surface coatings that release substances which can keep the implant free of bacteria until tissue integration is achieved. The use of chitosan in this study helped to prove this fact. Moreover, results of SEM reveal that there are stable and homogeneous layer of coating on the fabric surface. Though the proposed process is technochemically feasible, however, to implement it at a large industrial scale, there is a need to perform techno-economic and environmental impact study of the proposed method [44] and energy released study. A better understanding of the role of lateral forces can also help in future surface engineering of new surface modifications to resist bacterial adhesion.

\section{References}

[1] Hannan Safiyyah Tan Sian Hui Abdullah, Siti Nur Aqlili Riana Mohd Asseri, Mohamad WNKW, Kan S-Y, Azmi AA, Julius FSY, et al. Green synthesis, characterization and applications of silver nanoparticlemediated by the aqueous extract of red onion peel. Environmental Pollution. 2020:116295.

[2] Ponsanti K, Tangnorawich B, Ngernyuang N, Pechyen C. A flower shape-green synthesis and characterization of silver nanoparticles (AgNPs) with different starch as a reducing agent. Journal of Materials Research and Technology. 2020;9(5):11003-11012.

[3] Li Z, Lee D, Sheng X, Cohen RE, Rubner MF. Two-level antibacterial coating with both release-killing and contact-killing capabilities. Langmuir. 2006;22(24):9820-9823.

[4] Huang S, Wang J, Zhang Y, Yu Z, Qi C. Quaternized carboxymethyl chitosan-based silver nanoparticles hybrid: microwave-assisted synthesis, characterization and antibacterial activity. nanomaterials. 2016;6(6):118.

[5] Setua P, Chakraborty A, Seth D, Bhatta MU, Satyam PV, Sarkar N. Synthesis, optical properties, and surface enhanced raman scattering of silver nanoparticles in nonaqueous methanol reverse micelles. The Journal of Physical Chemistry C. 2007;111(10):3901-3907.

[6] Sambhy V, Macbride MM, Peterson BR, Sen A. Silver bromide nanoparticle/polymer composites: Dual action tunable antimicrobial materials. Journal of the American Chemical Society. 2006;128(30):9798-9808.

[7] Chowdhury NR, Macgregor-Ramiasa M, Zilm P, Majewski P, Vasilev K. 'Chocolate’ silver nanoparticles: Synthesis, antibacterial activity and cytotoxicity. Journal of Colloid and Interface Science. 2016;482:151158. 
[8] Wren AW, Hassanzadeh P, Placek LM, Keenan TJ, Coughlan A, BoutelleLR, et al. Silver nanoparticle coated bioactive glasses - Composites with Dex/CMC hydrogels: Characterization, solubility, and in vitro biological studies. Macromolecular Bioscience. 2015;15(8):1146-1158.

[9] Yun J, Lee DG. Silver nanoparticles: A novel antimicrobial agent. Antimicrobial Nanoarchitectonics. 2017:139-166.

[10] Wang Z, Sun Y, Wang D, Liu H, Boughton RI. In situ fabrication of silver nanoparticle-filled hydrogen titanate nanotube layer on metallic titanium surface for bacteriostatic and biocompatible implantation. International Journal of Nanomedicine. 2013:2903.

[11] Yuan X, Chen Y. Visual determination of $\mathrm{Cu} 2$ through copper-catalysed in situ formation of Ag nanoparticles. The Analyst. 2012;137(19):4516.

[12] Willner I, Baron R, Willner B. Growing metal nanoparticles by enzymes. Advanced Materials. 2006;18(9):1109-1120.

[13] Torabfam M, Yüce M. Microwave-assisted green synthesis of silver nanoparticles using dried extracts of Chlorella vulgaris and antibacterial activity studies. Green Processing and Synthesis. 2020;9(1):283-293.

[14] Li H-J, Zhang A-Q, Hu Y, Sui L, Qian D-J, Chen M. Large-scale synthesis and self-organization of silver nanoparticles with Tween 80 as a reductant and stabilizer. Nanoscale Research Letters. 2012;7(1):612.

[15] Mayoral A, Magen C, Jose-Yacaman M. High-yield production of long branched Au nanoparticles characterized by atomic resolution transmission electron microscopy. Crystal Growth \& Design. 2011;11(10):4538-4543.

[16] Bukhari SNA, Hussain MA, Shah A, Jantan I, Shah MR, Tahir MN, et al. Hydroxypropylcellulose as a novel green reservoir for the synthesis, stabilization, and storage of silver nanoparticles. International Journal of Nanomedicine. 2015:2079.

[17] Lou XW, Yuan C, Archer LA. An unusual example of hyperbranched metal nanocrystals and their shape evolution. Chemistry of Materials. 2006;18(17):3921-3923.

[18] Arcos AAA, Miranda-Hernández M. Electrochemical formation of silver nanoparticles and nanoclusters on multiwall carbon nanotube electrode films. Silver Nanoparticles - Fabrication, Characterization and Applications. 2018.

[19] Sherif HH, Khalil SK, Hegazi AG, Khalil WA, Moharram MA. Factors affecting the antibacterial activity of chitosan-silver nanocomposite. IET Nanobiotechnology. 2017;11(6):731-737.

[20] Choi J-Y, Ramachandran G, Kandlikar M. The impact of toxicity testing costs on nanomaterial regulation. Environmental Science \& Technology. 2009;43(9):3030-3034.

[21] Dayioglu H, Kut D, Merdan N, Canbolat S. The effect of dyeing properties of fixing agent and plasma treatment on silk fabric dyed with natural dye extract obtained from Sambucus Ebulus L. Plant. Procedia Social and Behavioral Sciences. 2015;195:1609-1617.

[22] Tayel AA, El-Tras WF, Abdel-Monem OA, El-Sabbagh SM, Alsohim AS, El-Refai EM. Production of anticandidal cotton textiles treated with oak gall extract. Revista Argentina de Microbiología. 2013;45(4):271-276.

[23] Thiry E, Addie D, Belák S, Boucraut-Baralon C, Egberink H, Frymus T, et al. Feline herpesvirus infection: ABCD guidelines on prevention and management. Journal of Feline Medicine and Surgery. 2009;11(7):547_ 555.

[24] Gedanken A, Perkas N, Perelshtein I. Coating antibacterial nanoparticles on textiles: Towards the future hospital in which all textiles will be antibacterial. Nano Online. 2016.

[25] Wang C, Lv J, Ren Y, Zhou Q, Chen J, Zhi T, et al. Cotton fabric with plasma pretreatment and $\mathrm{ZnO}$ /Carboxymethyl chitosan composite finishing for durable UV resistance and antibacterial property. Carbohydrate Polymers. 2016;138:106-113.

[26] Borkow G, Felix A, Gabbay J. Copper-impregnated antimicrobial textiles; an innovative weapon to fight infection. Medical and Healthcare Textiles. 2010:14-22.

[27] Klempner MS, Hu LT, Evans J, Schmid CH, Johnson GM, Trevino RP, et al. Two controlled trials of antibiotic treatment in patients with persistent symptoms and a history of lyme disease. New England Journal of Medicine. 2001;345(2):85-92.

[28] Knidri HE, Laajeb A, Lahsini A. Chitin and chitosan: chemistry, solubility, fiber formation, and their potential applications. Handbook of Chitin and Chitosan. 2020:35-57.

[29] Kasai D, Chougale R, Masti S, Chalannavar R, Malabadi RB, Gani R. Influence of Syzygium cumini leaves extract on morphological, thermal, mechanical, and antimicrobial properties of PVA and PVA/chitosanblend films. Journal of Applied Polymer Science. 2018;135(17):46188.

[30] Yang F, Yao J, Chen X, Shao Z. Preparation and antimicrobial properties Of Pva/tannin blend films. Acta Polymerica Sinica. 2012;012(2):125-130.

[31] Gedanken A, Perkas N, Perelshtein I. Coating antibacterial nanoparticles on textiles: Towards the future hospital in which all textiles will be antibacterial. nano Online. 2016; 
[32] Mahltig B, Bottcher H. Modified silica sol coatings for water-repellent textiles: Journal of Sol-Gel Science and Technology. 2003. 27(1): 43-52.

[33] Gao Y, Cranston R. Recent advances in antimicrobial treatments of textiles. Textile Research Journal. 2008;78(1):60-72.

[34] Yazdankhah SP, Scheie AA, Høiby EA, Lunestad B-T, Heir E, Fotland TØ, et al. Triclosan and antimicrobial resistance in bacteria: an overview. Microbial Drug Resistance. 2006;12(2):83-90.

[35] Rosu C, Lin H, Jiang L, Breedveld V, Hess DW. Sustainable and long-time 'rejuvenation' of biomimetic water-repellent silica coating on polyester fabrics induced by rough mechanical abrasion. Journal of Colloid and Interface Science. 2018;516:202-214.

[36] Ortelli S, Costa AL, Blosi M, Brunelli A, Badetti E, Bonetto A, et al. Colloidal characterization of CuO nanoparticles in biological and environmental media. Environmental Science: Nano. 2017;4(6):1264-1272.

[37] Abramova A, Gedanken A, Popov V, Ooi E-H, Mason TJ, Joyce EM, et al. A sonochemical technology for coating of textiles with antibacterial nanoparticles and equipment for its implementation. Materials Letters. 2013;96:121-124.

[38] Petkova P, Francesko A, Fernandes MM, Mendoza E, Perelshtein I, Gedanken A, et al. sonochemical coating of textiles with hybrid ZnO/Chitosan antimicrobial nanoparticles. ACS Applied Materials \& Interfaces. 2014;6(2):1164-1172.

[39] Água RBD, Branquinho R, Duarte MP, Maurício E, Fernando AL, Martins R, et al. Efficient coverage of $\mathrm{ZnO}$ nanoparticles on cotton fibres for antibacterial finishing using a rapid and low cost in situ synthesis. New Journal of Chemistry. 2018;42(2):1052-1060.

[40] Yazdankhah SP, Scheie AA, Høiby EA, Lunestad B-T, Heir E, Fotland TØ, et al. Triclosan and antimicrobial resistance in bacteria: an overview. Microbial Drug Resistance. 2006;12(2):83-90.

[41] Zhu J-J, Wang H, Xu S, Chen H-Y. Sonochemical Method for the preparation of monodisperse spherical and rectangular lead selenide nanoparticles. Langmuir. 2002;18(8):3306-3310.

[42] Qureyshi S. Silver nanoparticles mediated through green route using pyrus seed extract. Journal of Basic Applied Chemistry. 2016;6(1):1.

[43] Sajid Z, Zhang Y, Khan F. Process design and probabilistic economic risk analysis of bio-diesel production. Sustainable Production and Consumption. 2016;5:1-15.

[44] Sajid Z, Khan F, Zhang Y. Process simulation and life cycle analysis of biodiesel production. Renewable Energy. 2016;85:945-952.

(C) 2020 by the author(s). This work is licensed under a Creative Commons Attribution 4.0 International License (http://creativecommons.org/licenses/by/4.0/). Authors retain copyright of their work, with first publication rights granted to Tech Reviews Ltd. 